\title{
Minimal Ni isotope fractionation during core formation as revealed by super-liquidus experiments
}

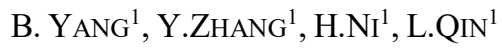

${ }^{1}$ CAS Key Laboratory of Crust-Mantle Materials and Environments, and CAS Center for Excellence in Comparative Planetology, School of Earth and Space Sciences, University of Science and Technology of China, Hefei 230026, China

Recently, the potential of $\mathrm{Ni}$ isotopic composition as a tracer for core formation has been explored. The isotope measurements on terrestrial samples and meteorites revealed a resolvable $\mathrm{Ni}$ isotope difference between the bulk silicate Earth and chondrites [1, 2]. This disagrees with experimental findings of negligible $\mathrm{Ni}$ isotope fractionation between the metal phase and the silicate phase at coreforming temperatures [3, 4]. However, the existing experiments were performed at sub-solidus conditions, whereas the metal-silicate separation during core formation is generally believed to occur in molten states. These experiments also suffered from several technical defects.

In this study we performed Ni-doped metal-silicate equilibrium experiments in a piston cylinder apparatus at $1873 \mathrm{~K}$ and $1 \mathrm{GPa}$ (super-liquidus condition) with run durations up to 8 hours. The Ni isotope compositions of the recovered samples were determined using a double-spike $\left({ }^{61} \mathrm{Ni}-{ }^{62} \mathrm{Ni}\right)$ method with MC-ICP-MS.

The silicate phases in our experimental products show notable enrichment in heavy $\mathrm{Ni}$ isotopes relative to the coexisting metal phases. However, the $\Delta^{60}$ Nisilicate-Metal value decreases with decreasing $\mathrm{Ni}$ content in the metal phase such that when we extrapolate the $\mathrm{Ni}$ content to $\sim 5 \mathrm{wt} . \%$ assuming a linear fitting, the $\Delta^{60} \mathrm{Ni}$ silicate-Metal value is found to be only $\sim 0.01 \pm 0.06 \%$ at $1873 \mathrm{~K}$. Therefore, our results suggest that core formation does not effectively change the $\mathrm{Ni}$ isotope composition of the silicate portion of the Earth and differentiated asteroids. The heterogeneity of Ni isotope compositions of different planetary reservoirs must arise from other processes, such as nebular condensation.

References: [1] Elliott, T., \& Steele, R. C. (2017) $R M G$, 82(1), 511-542. [2] Klaver, M. et al. (2020) GCA, 268, 405-421. [3] Lazar, C. et al. (2012) GCA, 86, 276-295. [4] Guignard, J. et al. (2020) GCA, 269, 238-256. 